\title{
Necrolytic erythema migrans in celiac disease
}

\section{Dassouli Ryme, Hanane BayBay, Souad Choukri, Zakia Douhi, Sara Elloudi, Fatima Zahra Mernissi}

\author{
Department of Dermatology, University Hospital Hassan II, Fès, Morocco
}

Corresponding author: Dassouli Ryme, MD, E-mail: dassouliryme@gmail.com

\begin{abstract}
Erythema migrans necrolytica is a red, blistering rash that spreads over the skin. It particularly affects the skin around the mouth and distal extremities, but can also be found on the lower abdomen, buttocks, perineum and groin. It is strongly associated with glucagonoma, a glucagon-producing tumor of the pancreas, but is also seen in a number of other conditions, including liver disease and intestinal malabsorption such as celiac disease. We present a case of a patient with a history of poorly followed celiac disease presenting with a clinical picture of ENM. This rare case adds to our understanding of the clinical presentation of NME, as well as highlights the importance of acting in a timely manner to avoid the most redoubtful complications.
\end{abstract}

Key words: Necrolytic erythema migrans; Celiac disease; Malabsorption; Pseudoglucagonoma

\section{INTRODUCTION}

Celiac disease is one of the inflammatory bowel diseases responsible for widespread malabsorption and nutritional deficiencies. Among the rare dermatological manifestations that it can cause is necrotic erythema migrans. The etiopathogenesis is still poorly understood, but it is probably due to vitamin, zinc and amino acid deficiencies. We report the case of a 17-year-old patient with necrotic erythema migrans during a relapse of celiac disease. Although the pathogenesis of necrotic erythema migrans remains unknown, global malabsorption and abrupt deficiencies appear to have played a causal role in the development of this dermatosis in our patient.

\section{CASE REPORT}

The case involved a 16-year-old boy of nonconsanguineous parents, known to have been diabetic for 3 years and on insulin with poor compliance, followed up for celiac disease since the age of 5 years, with acute diarrhea dating back one week following a deviation from the diet, and pruritic and painful skin eruptions of acute onset in the central part of the face and the perineum. The similar episodes recurred as soon as gluten was introduced into her diet, along with severe diarrhea, and regressed completely within a week after stopping gluten and following vitamin supplementation. The lesions started in the folds and then progressively spread to the rest of the body. The mother also reported a weight loss of $5 \mathrm{~kg}$ during one week with an alteration of the general state and feverish feelings are also reported. The general examination showed an altered patient, dehydrated and malnourished with a loss of muscle mass and hollow eyeballs associated with oedema of the lower limbs taking the cup. There was also a very obvious growth retardation. The dermatological examination found erosive placards surmounted by hemorrhagic crusts surrounded by an epidermal collar which were located at the peri-anal level at the level of the pubic region of the scrotum of the lower abdomen and of the internal faces of the thighs (Figs. la and $1 \mathrm{~b}$ ), one also noted a fissured angular dry cheilitis and a glossitis made of a partially depilated red tongue (Figs. 2a and 2b). The rest of the somatic examination found hepatosplenomegaly, abdominal bloating. Significant laboratory values included decreased albumin $(17 \mathrm{~g} / \mathrm{dl})$, increased bilirubin $(2.2 \mathrm{mg} / \mathrm{dl})$, hyperglycemia $(2.14 \mathrm{~g} / \mathrm{dl})$,

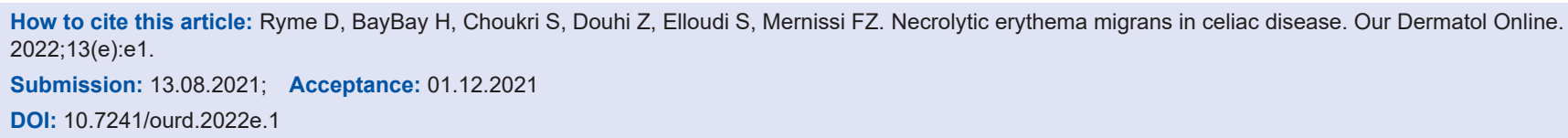




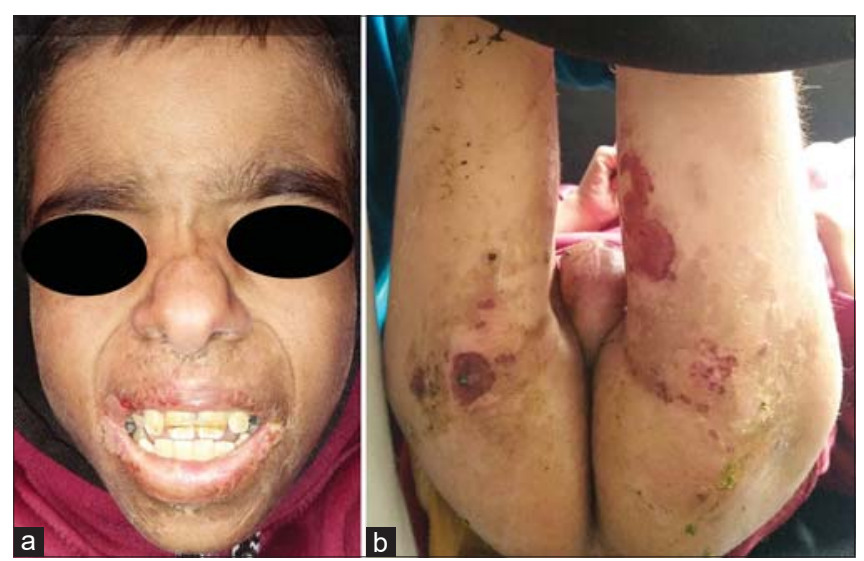

Figure 1: ( $a$ and b) Papillary glossitis with angular cheilitis in a cachectic patient.

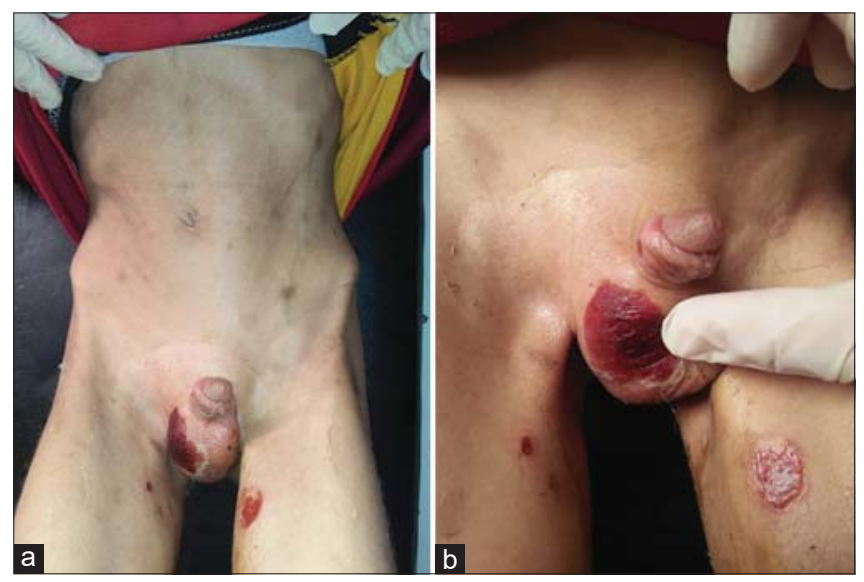

Figure 2: $(a$ and $b)$ Erosive placard surrounded by epidermal collar and surmounted by hemorrhagic crusts on the scrotum, perineum, inner thighs and buttocks.

microcytic anemia $(8.5 \mathrm{~g} / \mathrm{dl})$ with elevated transaminases and sedimentation rate. Lipasemia was normal and radiographic evaluation revealed bilateral effacement of the Douglas's pouch. There was no personal or family history of neuroendocrine tumor or background of glucagomnoma. Plasma glucagon levels were not obtained. The patient was dead because of septic shock. Although the plasma glucagon levels were not obtained, the clinical aspect of the lesions, their topography, the recurrent character following the deviation from the diet, the annular aspect described by the mother with a progressive extension and the spontaneous regression in one week following the vitamin supplementation and the avoidance of gluten pushed us to retain a migratory necrolytic erythema.

\section{DISCUSSION}

Becker et al [1] were the first to describe NME as a characteristic skin reaction pattern seen in the presence of pancreatic islet cell carcinoma. More recently, Kelly et al described NME in the absence of glucagonoma as the pseudoglucagonomia syndrome [2-4].

The most frequently observed conditions related to NME apart from pancreatic tumor are celiac disease or malabsorption syndrome in addition to zinc, attyric acid, and biotin deficiencies $[1,5,6]$, cirrhosis, malignancies, and pancreatitis [3]; less common conditions are hepatitis, inflammatory bowel disease, heroin abuse, and odontogenic abscess. In the case of a first flare of necrolytic erythema migrans, the search for an underlying glucagonoma is paramount. If this diagnosis is excluded, other pathologies accompanying necrolytic erythema migrans should be investigated, such as liver disease, malabsorption disorders, and non-insular tumors $[4,5]$.

Celiac disease (CD) can be defined as a chronic immune-mediated enteropathy dependent on gluten ingestion in genetically susceptible individuals. It is characterized by intestinal malabsorption and subtotal or total atrophy of the intestinal villi that improves after a gluten-free diet. It is manifested by diarrhea, weight loss, and nutritional deficiencies, including iron, folate, calcium, zinc, amino acids, and vitamin $\mathrm{D}[2,6]$. However, there is a wide variety of extraintestinal clinical presentations, including deficiency-related dermatologic manifestations such as porphyria, pellagra, and necrotic erythema migrans [2].

The dermatologic manifestations of necrotic erythema migrans are characterized by an annular, irregular eruption of papules, scaly erythematous plaques, and patches with superficial epidermal necrosis, central flaccid bullae, and crusted erosions. Lesions may be extensive but are usually localized to the inguinal areas, perineum, lower abdomen, thighs, and distal extremities $[5,6,7]$. They are often pruritic, painful, and prone to secondary infection that is difficult to manage. Angular cheilitis, glossitis, or alopecia may also be present. After healing of the lesions, residual areas of hyperpigmentation and a peripheral collar of scales may remain $[8,9]$.

There are many theories regarding the pathogenesis of ENM, including direct action of glucagon in inducing skin necrolysis, hypoaminoacidemia-induced epidermal protein deficiency and necrolysis, nutritional or metabolic deficiency of zinc or essential fatty acids, enteroglucagon induction of inflammatory 
mediators, and generalized malabsorption $[7,9,10]$. The occurrence of necrolytic erythema migrans in celiac disease reinforces current pathophysiological hypotheses suggesting a role for hypoprotidemia and plasma amino acid deficiency in necrolytic erythema migrans. In addition, glucagon-induced glycogenolytic and gluconeogenic effects may lead to depletion and necrolysis of epidermal proteins [10].

During necrolytic erythema migrans complicating untreated celiac disease, pancreatic glucagon levels are usually mildly elevated, but enteroglucagon levels are often greatly increased [2]. Reports have shown that in this case, treatment with a gluten-free diet not only resulted in resolution of malabsorption and improvement of small bowel histology, but was often accompanied by disappearance of necrolytic erythema migrans, normalization of plasma glucagon levels, and a marked reduction in the number of enteroglucagonproducing crypt cells $\left(0.2 / \mathrm{mm}^{2}\right.$ mucosa); $[2,10,11]$. This was the case in our patient whose lesions regressed after restoration of the diet, unfortunately the profound depletion and superinfection during this episode led to the death of our patient. Hence the importance of early intervention and supplementation with trace elements and zinc in celiac disease complicated by ENM.

\section{CONCLUSION}

In our case, we highlight that ENM could be caused by celiac disease, although it is rare. Early diagnosis and supplementation with vitamins and oligoelements as well as diet restoration is essential to avoid complications and dramatic consequences.

\section{Consent}

The examination of the patient was conducted according to the principles of the Declaration of Helsinki.
The authors certify that they have obtained all appropriate patient consent forms, in which the patients gave their consent for images and other clinical information to be included in the journal. The patients understand that their names and initials will not be published and due effort will be made to conceal their identity, but that anonymity cannot be guaranteed.

\section{REFERENCES}

1. Becker S, Kahn D, Rothman S. Cutaneous manifestations of internal malignant tumors. Arch Dermatol. 1942;45:1069-80.

2. Kelly CP, Johnston CF, Nolan N, Keeling PW, Weir DG. Necrolytic migratory erythema with elevated plasma enteroglucagon in celiac disease. Gastroenterology. 1989; 96:1350-3.

3. Liu JW, Qian YT, Ma DL. Necrolytic Migratory Erythema. JAMA Dermatol. 2019;155:1180.

4. Toberer F, Hartschuh W, Wiedemeyer K. Glucagonoma-associated necrolytic migratory erythema. Am J Dermatopathol. 2019; 41:29-32.

5. Tolliver S, Graham J, Kaffenberger BH. A review of cutaneous manifestations within glucagonoma syndrome: necrolytic migratory erythema. Int J Dermatol. 2018;57:642-5.

6. Bledsoe AC, King KS, Larson JJ, Snyder M, Absah I, et al. Micronutrient deficiencies are common in contemporary celiac disease despite lack of overt malabsorption symptoms. Mayo Clin Proc. 2019;94:1253-60.

7. Salaheldin Y, El Ansari W, Aljaloudi E, Elhag W. Third reported case of rare necrolytic migratory erythema associated with bacteraemia due to severe zinc deficiency after revisional Roux-En-Y gastric bypass: case report and literature review. Eat Weight Disord. 2021 Jun 1. doi: 10.1007/s40519-021-01154-z.

8. Bosch-Amate X, Riera-Monroig J, Iranzo Fernández P. Glucagonoma-related necrolytic migratory erythema. Med Clin. 2020;155:418-9.

9. He S, Zeng W, Geng S, Jia J. Glucagonoma syndrome with atypical necrolytic migratory erythema. Indian J Dermatol Venereol Leprol. 2021;87:49-53.

10. Mourguet M, Lajaunie R, Schouler M, Godart M, Bonnet D, Riffaud L, et al. Facial edema and erythroderma in a 54 year-old woman]. Rev Med Interne. 2020;41:210-3

11. Luber AJ, Ackerman LS, Culpepper KS, Buschmann CM, Koep LJ. Paediatric necrolytic migratory erythema as a presenting sign of glucagonoma syndrome. Br J Dermatol. 2016;174:1092-5.

Copyright by Dassouli Ryme, et al. This is an open access article distributed under the terms of the Creative Commons Attribution License, which permits unrestricted use, distribution, and reproduction in any medium, provided the original author and source are credited.

Source of Support: Nil, Conflict of Interest: None declared. 